\title{
CONSTRUCTING CATEGORIES AND SETOIDS OF SETOIDS IN TYPE THEORY
}

\author{
ERIK PALMGREN $^{a}$ AND OLOV WILANDER $^{b}$ \\ ${ }^{a}$ Stockholm University, Department of Mathematics, 10691 Stockholm, Sweden. \\ e-mail address: palmgren@math.su.se \\ ${ }^{b}$ Stockholm University, Department of Mathematics. Current affiliation: Sjöland \& Thyselius, Box \\ 6238, 10234 Stockholm. \\ e-mail address: olov.wilander@st.se
}

\begin{abstract}
In this paper we consider the problem of building rich categories of setoids, in standard intensional Martin-Löf type theory (MLTT), and in particular how to handle the problem of equality on objects in this context. Any (proof-irrelevant) family $F$ of setoids over a setoid $A$ gives rise to a category $\mathcal{C}(A, F)$ of setoids with objects $A$. We may regard the family $F$ as a setoid of setoids, and a crucial issue in this article is to construct rich or large enough such families. Depending on closure conditions of $F$, the category $\mathcal{C}(A, F)$ has corresponding categorical constructions. We exemplify this with finite limits. A very large family $F$ may be obtained from Aczel's model construction of CZF in type theory. It is proved that the category so obtained is isomorphic to the internal category of sets in this model. Set theory can thus establish (categorical) properties of $\mathcal{C}(A, F)$ which may be used in type theory. We also show that Aczel's model construction may be extended to include the elements of any setoid as atoms or urelements. As a byproduct we obtain a natural extension of CZF, adding atoms. This extension, CZFU, is validated by the extended model. The main theorems of the paper have been checked in the proof assistant Coq which is based on MLTT. A possible application of this development is to integrate set-theoretic and type-theoretic reasoning in proof assistants.
\end{abstract}

\section{INTRODUCTION}

Martin-Löf type theory (MLTT) and its manifestations, in proof assistants such as Agda and Coq, is intended to be a framework for formalizing (constructive) mathematics on a full scale. It is known that the intensional version of MLTT is sometimes difficult to employ when formalizing mathematics that depends on having (propositional) equality between sets or setoids. This may be troublesome in parts of category theory [15, 20] where an equality on objects is a standard assumption. A typical situation is when we wish to deal with some category of sets or setoids on equal footing to other categories. The built-in

2012 ACM CCS: [Theory of computation]: Logic; [Software and its engineering]: Software notations and tools - General programming languages - Language types-Functional languages.

Key words and phrases: Dependent type theory, setoids, formalization, categories, constructive set theory.

${ }^{b}$ This work was supported by a grant (Dnr 621-2008-5076) from the Swedish Research Council (VR).

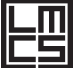

GICAL METHODS IN COMPUTER SCIENCE
DOI:10.2168/LMCS-10(3:25)2014

C) E. Palmgren and O. Wilander (c) Creative Commons 
propositional equalities of type theory, given by the intensional identity types, are not extensional enough for this work without further complications. The root of the problem is that the intensional identity type of MLTT induces a non-trivial groupoid structure on types [8]. This can be avoided by introducing extra elimination axioms like the K-axiom of Streicher [18, the Uniqueness of Identity Proofs axiom, or weaker axioms [20]. Adding these axioms is, however, an unsatisfactory solution according to the general philosophy of MLTT, where the elimination rule is supposed to be generated by the introduction rule.

In this paper we consider solutions to this problem within the standard intensional version of MLTT with one universe and W-types. In Section 4.1 we recall that any (proofirrelevant) family $F$ of setoids over a setoid $A$ gives rise to a category $\mathcal{C}(A, F)$ of setoids with objects $A$. We may regard the family $F$ as a setoid of setoids, and a crucial issue here is to construct rich or large enough such families. Depending on closure conditions of $F$, the category $\mathcal{C}(A, F)$ has corresponding categorical constructions. A first solution is to explicitly construct $F$ such that it is closed under particular constructions, here exemplified by finite limits (Section 4.2). A smoother solution is however to employ a universe $V$ of iterative sets that forms a model of Aczel-Myhill constructive set theory (CZF), and consider the category of setoids that the sets of $V$ induces. This turns out to be a well-behaved category of setoids, which is isomorphic to the internal category of sets of $V$ (Theorem 5.5). Theorem 5.5 allows passage between the setoids of type theory and the sets of $V$. The model and the theorem have been formalized in the proof assistant Coq, and give, in principle, a method for formalizing further category-theoretic results in Coq that depend on a good category of sets. This makes it possible to integrate set-theoretic and type-theoretic reasoning, where set theory has a simpler language which is better adapted to solve certain complications arising from transport functions of families of setoids, and type theory has a more direct computational interpretation.

Models of CZF have previously been implemented in systems similar to Coq: in LEGO by Mendler [12] and in Agda/Alfa by Takeyama [19]. See also Hickey [6] and Yu [21] for work done in MetaPRL. However, we add a new twist here by allowing urelements or atoms in the model, and importantly, giving the relation to setoids, via the notion of a $V$-representable setoid (Section 5.2). Our formalized model moreover allows us to embed an arbitrary setoid $M$ in a CZF-universe $V(M)$. As a bonus of the construction $V(M)$, we obtain a model of CZF with atoms (elements of $M$ ), which is formalized as a first-order theory CZFU (Section 5.4). We end by some remarks on the formalization in Coq (Section 6).

\section{SETOIDS}

In the following we freely use the propositions-as-types principle in the logical arguments. Thus we may speak of a proof $q$ of a proposition $Q$, meaning that $q$ is an element of type $Q$ which is written $q: Q$ as usual. In our implementation in Coq this corresponds to avoiding the built-in type Prop and using Set or Type for propositions. (See Section 6])

Recall that a setoid $A=\left(|A|,=_{A}\right)$ is a type $|A|$ with an equivalence relation ${ }_{A}$. We denote the constructions associated with proofs of reflexivity, symmetry and transitivity as follows

$$
\begin{aligned}
& \operatorname{ref}(x): x={ }_{A} x \quad(x:|A|) \\
& p^{-1}: y={ }_{A} x \quad\left(x, y:|A|, p: x={ }_{A} y\right) \\
& q \circ p: x={ }_{A} z \quad\left(x, y, z:|A|, p: x={ }_{A} y, q: y==_{A} z\right)
\end{aligned}
$$


We shall often write $x \in A$ for $x:|A|$ to simplify notation. For setoids $A$ and $B$, an extensional function $f: A \longrightarrow B$ is a pair $f=\left(|f|\right.$, $\left.x t_{f}\right)$ where $|f|:|A| \longrightarrow|B|$ and $\operatorname{ext}_{f}$ is a proof-object for extensionality of the operation $|f|$, that is that

$$
(\forall x, y \in A)\left(x={ }_{A} y \Longrightarrow|f|(x)={ }_{B}|f|(y)\right) .
$$

We write $f(x)$ for $|f|(x)$.

For setoids $A$ and $B$ denote by $\operatorname{Ext}(A, B)$ the setoid of extensional functions from $A$ to $B$, with point-wise equality $\left(=_{\text {ext }}\right)$ as equivalence relation. The setoids and extensional functions form an E-category, which shall be named Setoids here. We recall that an Ecategory $\mathcal{C}$ has a type of objects with no equality assumed between them. The morphisms, denoted $\operatorname{Hom}_{\mathcal{C}}(A, B)$, from object $A$ to $B$ is a setoid and the composition operation

$$
\circ: \operatorname{Hom}_{\mathcal{C}}(B, C) \times \operatorname{Hom}_{\mathcal{C}}(A, B) \longrightarrow \operatorname{Hom}_{\mathcal{C}}(A, C)
$$

is an extensional function. The usual laws for composition and identity are supposed to be satisfied.

Example 2.1. Let $F(x)(x: S)$ be a family of setoids indexed by a type $S$. Then an E-category $\mathcal{E}(S, F)=\mathcal{E}$ of setoids can be formed whose type of objects is $S$ and where

$$
\operatorname{Hom}_{\mathcal{E}}(a, b)=\operatorname{Ext}(F(a), F(b)) .
$$

Remark 2.2. The E-category Setoids considered here (and elsewhere) is closely related to the exact completion of a syntactic category arising from type theory (cf. [5]). The match is not precise since the E-category is internal to type theory, whereas the exact completion takes place on the meta-level. Barthe et al. [3] compare several variants of the notion of setoid and their formalization in Coq. In their terminology we use total setoids but employ Set-valued equivalence relations instead of their Prop-valued ones.

\section{FAMILIES OF SETOIDS}

A good notion of a family of setoids over a setoids is the following (compare the discussion in [15]). A proof irrelevant family $F$ of setoids over a setoid $A$ - or just family of setoids - consists of a setoid $F(x)=\left(|F(x)|,=_{F(x)}\right)$ for each $x \in A$, and for $p:\left(x==_{A} y\right)$ an extensional function $F(p) \in \operatorname{Ext}(F(x), F(y))$ which satisfies the conditions (F1) - (F3) below.

(F1) $F(\operatorname{ref}(x))=$ ext $\operatorname{id}_{F(x)}$ for $x \in A$.

(F2) $F(p)={ }_{\text {ext }} F(q)$ for $p, q: x={ }_{A} y$ and $x, y \in A$. This is the proof-irrelevance condition, since $F(p)$ does not depend on $p$.

(F3) $F(q) \circ F(p)={ }_{\text {ext }} F(q \circ p)$ for $p: x={ }_{A} y, q: y={ }_{A} z$ and $x, y, z \in A$.

The function $F(p)$ is sometimes called a transport function. Because of condition (F2), condition (F1) can be replaced by (F1')

$$
(\forall x \in A)\left(\forall p: x={ }_{A} x\right) F(p)={ }_{\text {ext }} \operatorname{id}_{F(x)}
$$

and condition (F3) can be replaced by (F3')

$$
(\forall x, y, z \in A)\left(\forall p: x={ }_{A} y\right)\left(\forall q: y={ }_{A} z\right)\left(\forall r: x={ }_{A} z\right) F(q) \circ F(p)={ }_{\mathrm{ext}} F(r) .
$$

We shall sometimes use the notation $x \cdot p$ for $F(p)(x)$ when $F$ is clear from the context.

As can be seen from (F1) - (F3) a family $F$ may be regarded as a functor (or rather E-functor) from the discrete E-category $A^{\#}$, induced by $A$, to Setoids. 


\section{From families to CAtegories of Setoids}

It is well-known that the E-category of setoids in Martin-Löf type theory forms a locally cartesian closed (LCC) category; see [7]. It can moreover be shown to be a pretopos with further properties [13. In fact, one can straightforwardly verify in Coq (see for instance [17) that the E-category of setoids forms an LCC pretopos. For categories of setoids with equality on objects the constructions are more delicate and this is the subject of this and the next section.

Categories can be presented in an essentially algebraic way; cf. 10. This is a useful formulation especial for doing category theory internally to a category, but also in set theory. A (small) category $\mathcal{C}$ is a triple of classes (sets) $\mathcal{C}_{0}, \mathcal{C}_{1}, \mathcal{C}_{2}$ consisting of objects, arrows and composable arrows, equipped with class functions (functions) id $: \mathcal{C}_{0} \longrightarrow \mathcal{C}_{1}$, dom, cod : $\mathcal{C}_{1} \longrightarrow \mathcal{C}_{0}$ and $\mathrm{cmp}$, fst, snd : $\mathcal{C}_{2} \longrightarrow \mathcal{C}_{1}$ that satisfy the axioms

1. $\operatorname{dom}(\operatorname{id}(x))=x$,

2. $\operatorname{cod}(\operatorname{id}(x))=x$,

3. $\operatorname{dom}(\mathrm{cmp}(u))=\operatorname{dom}(\mathrm{fst}(u))$,

4. $\operatorname{cod}(\mathrm{cmp}(u))=\operatorname{cod}(\operatorname{snd}(u))$,

$4 \frac{1}{2} \cdot \operatorname{cod}(\operatorname{fst}(u))=\operatorname{dom}(\operatorname{snd}(u))$,

and

5. $\mathrm{fst}(u)=\operatorname{fst}(v), \operatorname{snd}(u)=\operatorname{snd}(v) \Longrightarrow u=v$,

6. $\operatorname{dom}(f)=\operatorname{cod}(g) \Longrightarrow \exists u \in \mathcal{C}_{2}(\operatorname{snd}(u)=f \wedge \mathrm{fst}(u)=g)$,

7. $\mathrm{fst}(u)=\mathrm{id}(y) \Longrightarrow \operatorname{cmp}(u)=\operatorname{snd}(u)$,

8. $\operatorname{snd}(u)=\mathrm{id}(x) \Longrightarrow \operatorname{cmp}(u)=\operatorname{fst}(u)$,

9. $\mathrm{fst}(w)=\mathrm{fst}(v), \operatorname{snd}(v)=\mathrm{fst}(u), \operatorname{snd}(u)=\operatorname{snd}(z), \operatorname{snd}(w)=\operatorname{cmp}(u), \operatorname{cmp}(v)=\mathrm{fst}(z) \Longrightarrow$ $\operatorname{cmp}(w)=\operatorname{cmp}(z)$.

The category Set of sets and functions can be constructed in the standard way in ZF or CZF using these operations, and its categorical properties developed, following e.g. [4].

We can simply obtain a type-theoretic definition by replacing sets and classes with setoids (with respective size restrictions) and functions by extensional functions.

A functor $F: \mathcal{B} \longrightarrow \mathcal{C}$ is a triple of extensional functions $F_{k}: \mathcal{B}_{k} \longrightarrow \mathcal{C}_{k}, k=0,1,2$, such that all operations of the categories are preserved, that is

$$
\begin{aligned}
& F_{1} \circ \text { id }=\text { id } \circ F_{0}, \\
& F_{0} \circ \text { dom }=\text { dom } \circ F_{1}, \\
& F_{0} \circ \mathrm{cod}=\operatorname{cod} \circ F_{1}, \\
& F_{1} \circ \text { fst }=\text { fst } \circ F_{2}, \\
& F_{1} \circ \mathrm{snd}=\operatorname{snd} \circ F_{2}, \\
& F_{1} \circ \mathrm{cmp}=\mathrm{cmp} \circ F_{2} .
\end{aligned}
$$

The axioms 1 - 9 take a more familiar form if we rewrite them using the composition predicate $\operatorname{Comp}(f, g, h)$ (or $f \circ g \equiv h$ ) defined by

$$
\left(\exists u \in \mathcal{C}_{2}\right)(\text { fst }(u)=g \wedge \operatorname{snd}(u)=f \wedge \mathrm{cmp}(u)=h) .
$$

Remark 4.1. Any category $\mathcal{C}$ may be viewed as an E-category $\overline{\mathcal{C}}$ by ignoring the equality on objects and defining $\operatorname{Hom}_{\overline{\mathcal{C}}}(a, b)$ to be the setoid

$$
\left(\left(\Sigma f \in \mathcal{C}_{1}\right)[\operatorname{dom}(f)=a \wedge \operatorname{cod}(f)=b], \sim\right)
$$

where $(f, p) \sim\left(f^{\prime}, p\right)$ iff $f=\mathcal{C}_{1} f^{\prime}$. Composition and identity are then defined in the obvious way using the axioms above. 
We may even consider Hom as a proof-irrelevant family over the setoid $\mathcal{C}_{0} \times \mathcal{C}_{0}$. This gives an equivalent notion of category which is perhaps more natural in the type-theoretic language.

4.1. Construction of a category of setoids. We recall the following construction from [15] and [20]. Any family $F$ of setoids over a setoid $A$ gives rise to a category of setoids $\mathcal{C}=\mathcal{C}(A, F)$ in the following way. The objects are given by the index setoid $\mathcal{C}_{0}=A$, and are thus equipped with equality, and the setoid of arrows $\mathcal{C}_{1}$ is

$$
((\Sigma x, y:|A|) \operatorname{Ext}(F(x), F(y)), \sim)
$$

where two arrows are equal $(x, y, f) \sim(u, v, g)$ if, and only if, there are proof objects $p: x={ }_{A} u$ and $q: y={ }_{A} v$ such that the diagram

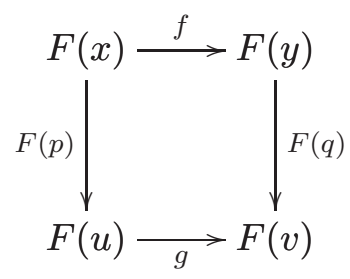

commutes, or equivalently

$$
(\forall t \in F(x))\left[f(t) \cdot q={ }_{F(v)} g(t \cdot p)\right] .
$$

(Note that $F(p)$ and $F(q)$ are independent of $p$ and $q$.) The domain and codomain maps dom : $\mathcal{C}_{1} \rightarrow \mathcal{C}_{0}$ and cod : $\mathcal{C}_{1} \rightarrow \mathcal{C}_{0}$ are given by $\operatorname{dom}(x, y, f)=x$ and $\operatorname{cod}(x, y, f)=y$. The setoid $\mathcal{C}_{2}$ of composable maps is then

$$
\left(\left(\Sigma h, k:\left|\mathcal{C}_{1}\right|\right)\left[\operatorname{cod}(h)=\mathcal{C}_{0} \operatorname{dom}(k)\right], \approx\right)
$$

where $(h, k, p) \approx\left(h^{\prime}, k^{\prime}, p^{\prime}\right)$ if and only if $h \sim h^{\prime}$ and $k \sim k^{\prime}$. The composition map $\mathrm{cmp}: \mathcal{C}_{2} \longrightarrow \mathcal{C}_{1}$ is given by

$$
\operatorname{cmp}((x, y, f),(u, v, g), p)=_{\operatorname{def}}(x, v, g \circ F(p) \circ f) .
$$

Furthermore, let

$$
\operatorname{fst}((x, y, f),(u, v, g), p)=_{\text {def }}(x, y, f) \quad \operatorname{snd}((x, y, f),(u, v, g), p)=_{\operatorname{def}}(u, v, g) .
$$

It is straightforward to verify

Theorem 4.2. If $F$ is a family of setoids over a setoid $A$, then $\mathcal{C}=\mathcal{C}(A, F)$ is a small category.

Lemma 4.3. In the category $\mathcal{C}(A, F)$ the composition predicate Comp may be characterized as follows

$$
\operatorname{Comp}((c, d, g),(a, b, f), \mathbf{h}) \Longleftrightarrow\left(\exists r: b={ }_{A} c\right)(a, d, g \circ F(r) \circ f) \sim \mathbf{h} .
$$

If $b$ and $c$ are definitionally equal, then $F(r)$ is the identity map. 
4.2. Closure conditions on families and categories. An important property of the category of sets is that the terminal object (i.e. the singleton set) generates the category. In such categories it is possible to interpret the internal logic in terms of elements; see [16]. We recall some definitions before proving that $\mathcal{C}(A, F)$ has the same property.

Let $\mathcal{D}$ be a category with terminal object 1 . Recall that an element of an object $X$ is an arrow $x: 1 \longrightarrow X$. An arrow $f: X \longrightarrow Y$ of the category is here called onto if for every $y: 1 \longrightarrow Y$, there is some $x: 1 \longrightarrow X$ with $f \circ x=y$. The arrow is as usual mono if for any $g, h: U \longrightarrow X$ in $\mathcal{D}, f g=f h$ implies $g=h$. If each arrow $f: X \longrightarrow Y$ in $\mathcal{D}$ that is both onto and mono, is also an isomorphism, then we say that 1 is a strong generator for $\mathcal{D}$. For a family $F$ of setoids over $A$, we say that $c \in A$ represents a setoid $C$ if $F(c)$ is isomorphic to $C$. We also say that $F$ contains $C$ (up to isomorphism). The category $\mathcal{C}(A, F)$ has a strong generator whenever the family $F$ contains the terminal object. This follows from the straightforwardly proven result. Note that part (d) uses the type-theoretic choice principle coming from $\Sigma$-elimination.

Proposition 4.4. Let $F$ be a family of setoids indexed by the setoid $A$, and suppose that $c \in A$ represents the terminal setoid. Then

(a) $c$ is the terminal object in $\mathcal{C}(A, F)$.

(b) If $(a, b, f)$ is an arrow of $\mathcal{C}(A, F)$ then it is mono if and only if $f: F(a) \longrightarrow F(b)$ is injective.

(c) If $(a, b, f)$ is an arrow of $\mathcal{C}(A, F)$ then it is onto if and only if $f: F(a) \longrightarrow F(b)$ is surjective.

(d) The terminal object of $\mathcal{C}(A, F)$ is a strong generator for the category.

If the family $F$ is a universe, we get a category $\mathcal{C}(A, F)$ with closure conditions depending on the type-theoretic closure conditions of the universe. In [13] it was shown that by letting $A, F$ be a particular universe of $U$-small setoids, the category is a locally cartesian closed pretopos with $W$. By a such a universe we mean that, for each $a \in A, F(a)$ is a setoid where both the underlying type $|F(a)|$ and the truth-values of $x={ }_{F(a)} y$ are in the type theoretic universe $U$. However, the construction of $A$ and $F$ in that paper used constructions going outside standard intensional type theory, in fact, a tacit assumption was made of a principle (see [15. Theorem 5.2]) which is equivalent to Uniqueness of Identity Proofs, which, in turn, is false in the groupoid model. In [20] a somewhat weaker axiom is proposed, which may possibly let the constructions of [13] go through. We have constructed (in Coq) a graded universe of setoids $A_{\omega}, F_{\omega}$, with no transfinite types, but closed under grade bounded $\Pi$ and $\Sigma$, as well as sums and coequalizers, to be able to mimic constructions of categorical universes in extensional type theory [11. However the expected categorical properties of $\mathcal{C}\left(A_{\omega}, F_{\omega}\right)$ have turned out quite difficult to verify formally. In the next subsection we present instead a method to taylor particular categorical universes which is more manageable.

4.3. Direct construction of categorical universes. We present a method for constructing categories of setoids closed under particular constructions, and exemplify with the construction of pullbacks.

Let $S$ be the inductive type defined by the rules

$$
\overline{\mathrm{b}: S} \quad \frac{i: S \quad j: S \quad k: S}{\mathrm{p}(i, j, k): S} .
$$


The identity on this type is decidable and satisfies

$$
I\left(S, \mathrm{p}(i, j, k), \mathrm{p}\left(i^{\prime}, j^{\prime}, k^{\prime}\right)\right) \Longleftrightarrow I\left(S, i, i^{\prime}\right) \wedge I\left(S, j, j^{\prime}\right) \wedge I\left(S, k, k^{\prime}\right) .
$$

This forms the setoid of construction stages. The symbol b signifies the basic stage.

Let $G$ be any family of setoids indexed by a setoid $B$. Then define by recursion on $s \in S, A_{s}$ and $F_{s}$, such that $A_{s}$ is a setoid and $F_{s}$ is family of setoids on $A_{s}$.

Let $A_{\mathrm{b}}=B$ and $F_{\mathrm{b}}=G$.

Let

$$
\begin{aligned}
A_{\mathrm{p}(i, j, k)}=\left(\left(\Sigma a: A_{i}\right)\left(\Sigma b: A_{j}\right)\left(\Sigma c: A_{k}\right)\left(\Sigma d: A_{k}\right)\right. & \\
& {\left.\left[\left(c={ }_{A_{i}} d\right) \times\left(F_{i}(a) \longrightarrow F_{k}(c)\right) \times\left(F_{j}(b) \longrightarrow F_{k}(d)\right)\right], \sim\right) }
\end{aligned}
$$

where

$$
(a, b, c, d, q, f, g) \sim\left(a^{\prime}, b^{\prime}, c^{\prime}, d^{\prime}, q^{\prime}, f^{\prime}, g^{\prime}\right)
$$

is given by

$$
\begin{gathered}
\left(\exists p_{1}: a={ }_{A_{i}} a^{\prime}\right)\left(\exists p_{2}: b={ }_{A_{j}} b^{\prime}\right)\left(\exists p_{3}: c={ }_{A_{k}} c^{\prime}\right)\left(\exists p_{4}: d={ }_{A_{k}} d^{\prime}\right) \\
F_{k}\left(p_{3}\right) \circ f=f^{\prime} \circ F_{i}\left(p_{1}\right) \wedge F_{k}\left(p_{4}\right) \circ g=g^{\prime} \circ F_{j}\left(p_{2}\right)
\end{gathered}
$$

and then

$$
\left.F_{\mathrm{p}(i, j, k)}(a, b, c, d, p, f, g)=\left(\left(\Sigma x: F_{i}(a)\right)\left(\Sigma y: F_{j}(b)\right)\left[F_{k}(p)(f(x))=_{F_{k}(d)} g(y)\right)\right], \approx\right) .
$$

where

$$
(x, y, r) \approx\left(x^{\prime}, y^{\prime}, r^{\prime}\right) \Longleftrightarrow{ }_{\text {def }} x={ }_{F_{i}(a)} x^{\prime} \wedge y={ }_{F_{j}(b)} y^{\prime} .
$$

For $\left(p_{1}, p_{2}, p_{3}, p_{4}, q_{1}, q_{2}\right):(a, b, c, d, p, f, g) \sim\left(a^{\prime}, b^{\prime}, c^{\prime}, d^{\prime}, p^{\prime}, f^{\prime}, g^{\prime}\right)$, define

$$
F_{\mathrm{p}(i, j, k)}\left(p_{1}, p_{2}, p_{3}, p_{4}, q_{1}, q_{2}\right): F_{\mathrm{p}(i, j, k)}(a, b, c, d, p, f, g) \longrightarrow F_{\mathrm{p}(i, j, k)}\left(a^{\prime}, b^{\prime}, c^{\prime}, d^{\prime}, p^{\prime}, f^{\prime}, g^{\prime}\right)
$$

by letting

$$
F_{\mathrm{p}(i, j, k)}\left(p_{1}, p_{2}, p_{3}, p_{4}, q_{1}, q_{2}\right)(x, y, r)=\left(F_{i}\left(p_{1}\right)(x), F_{j}\left(p_{2}\right)(y), r^{\prime}\right)
$$

where $r^{\prime}$ is some proof of

$$
F_{k}\left(p^{\prime}\right)\left(f^{\prime}\left(F_{i}\left(p_{1}\right)(x)\right)\right)={ }_{F_{k}\left(d^{\prime}\right)} g^{\prime}\left(F_{j}\left(p_{2}\right)(y)\right)
$$

(that can be obtained from (4.4)). It is straightforward to check that $F_{\mathrm{p}(i, j, k)}$ is a family of setoids over $A_{\mathrm{p}(i, j, k)}$. Moreover the following is a pullback square in the E-category of setoids

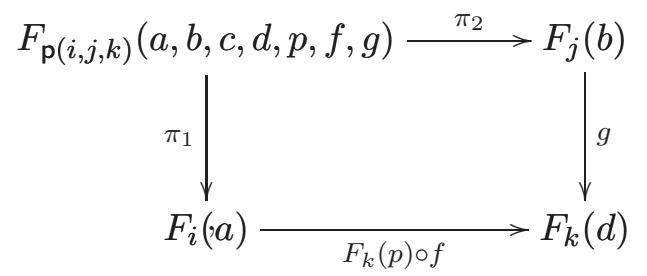

where $\pi_{1}(x, y, r)=x$ and $\pi_{2}(x, y, r)=y$.

Define using $I$-elimination, for $p: I\left(A, s, s^{\prime}\right)$,

$$
\operatorname{transport}_{\lambda s . A_{s}}(p): A_{s} \longrightarrow A_{s^{\prime}}
$$

by letting $C\left(s, s^{\prime}, p\right)={ }_{\operatorname{def}} A_{s} \longrightarrow A_{s^{\prime}}$ and transport $\lambda_{s . A_{s}}(\operatorname{ref}(s))=\lambda x: A_{s} \cdot x$.

Finally we define

$$
A_{\omega}=\left((\Sigma s: S) A_{s},{ }_{\omega}\right)
$$


where

$$
(s, a)={ }_{\omega}\left(s^{\prime}, a^{\prime}\right) \Longleftrightarrow_{\text {def }}\left(\exists p: I\left(S, s, s^{\prime}\right)\right) \text { transport }{ }_{\lambda s \cdot A_{s}}(p)(a)=_{A_{s^{\prime}}} a^{\prime}
$$

and

$$
F_{\omega}(s, a)=F_{s}(a)
$$

and further for $(p, q):(s, a)={ }_{\omega}\left(s^{\prime}, a^{\prime}\right)$, we define

$$
F_{\omega}(p, q)(x)=F_{s^{\prime}}(q)\left(H_{s, s^{\prime}, p}(x)\right),
$$

where $H_{s, s^{\prime}, p}: F_{s}(a) \longrightarrow F_{s^{\prime}}\left(\operatorname{transport}_{\lambda s . A_{s}}(p)(a)\right)$ is obtained by $I$-elimination with

$$
C\left(s, s^{\prime}, p\right)=_{\text {def }} F_{s}(a) \longrightarrow F_{s^{\prime}}\left(\operatorname{transport}_{\lambda s . A_{s}}(p)(a)\right)
$$

and $H_{s, s, \text { ref }}(s)=\lambda x: F_{s}(a) . x$. The identity type of $S$ is decidable, so it enjoys the Uniqueness of Identity Proofs property by Hedberg's theorem [15. Then one may easily verify that $F_{\omega}$ is a family of setoids over $A_{\omega}$. Furthermore the category $\mathcal{C}=\mathcal{C}\left(A_{\omega}, F_{\omega}\right)$ given by this family has chosen pullbacks, which means that there are two extensional functions $\mathrm{p}_{1}, \mathrm{p}_{2}: \mathrm{M}(\mathcal{C}) \longrightarrow \mathrm{Ob}(\mathcal{C})$ defined on the setoid of arrows with common codomain

$$
\mathrm{M}(\mathcal{C})=\left\{(f, g) \in \operatorname{Arr}(\mathcal{C})^{2}: \operatorname{cod}(f)=\operatorname{Ob}(\mathcal{C}) \operatorname{cod}(g)\right\}
$$

such that for all $(f, g) \in \mathrm{M}(\mathcal{C})$,

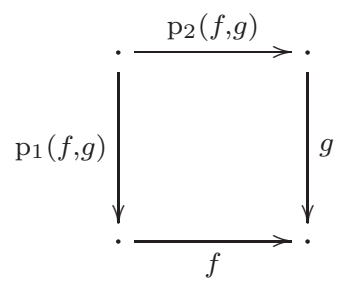

is a pullback. Using these constructions it is now possible to verify:

Theorem 4.5. The category $\mathcal{C}\left(A_{\omega}, F_{\omega}\right)$ has chosen pullbacks.

We expect that it should be possible to extend the construction above to other properties (e.g. LCC pretoposes) by adding new construction stages to (4.1). The formal verification will probably be quite cumbersome. However it is possible to obtain rich categorical universes that are smoother to construct and verify. In the next section we show that chosing $A$ and $F$ to be induced by the Aczel universe $V$ of iterative sets, the category $\mathcal{C}(A, F)$ gets good categorical properties; see Theorem [5.5.

\section{ACZEL'S ITERATIVE SETS AND SETOIDS}

It is known that the category of sets inside Constructive Zermelo-Fraenkel set theory (CZF) has good category-theoretic properties [4]. These can preferably be established on basis of the essentially algebraic formulation of categories given in Section 4. Aczel [1] presented a model of CZF in MLTT. This suggests that we may use such models of CZF to build useful categories for type theory. The model builds on the iterative conception of set, which is to say, a set is a, possibly infinite, well-founded tree, and where equality of sets is defined in terms of bisimulation. 
5.1. Iterative sets with urelements. We consider here a modification of Aczel's standard model of CZF, to be able to add urelements or atoms. For a universe $U, T(\cdot)$, and a setoid $M=\left(|M|,=_{M}\right)$ (of urelements), the set-theoretic universe $V(M)=V$ is inductively defined by the rules

$$
\frac{a: U \quad f: T(a) \longrightarrow V}{\sup (a, f): V} \quad \frac{b:|M|}{\operatorname{atom}(b): V} .
$$

The equality $=_{V}$ is the smallest relation satisfying the two rules

$$
\begin{gathered}
\frac{\forall x: T(a) \cdot \exists y: T(b) \cdot f(x)=_{V} g(y) \quad \forall y: T(b) \cdot \exists x: T(a) \cdot f(x)={ }_{V} g(y)}{\sup (a, f)={ }_{V} \sup (b, g)} \\
\frac{a={ }_{M} b}{\operatorname{atom}(a)={ }_{V} \operatorname{atom}(b)} .
\end{gathered}
$$

The membership relation is defined by

$$
u \in_{V} \sup (a, f) \Longleftrightarrow \exists x: T(a) \cdot u={ }_{V} f(x)
$$

and declaring $u \in_{V}$ atom $(b)$ to be false. We have $a={ }_{M} b$ iff atom $(a)=_{V}$ atom $(b)$, so that equality of atoms is exactly that of the setoid. The standard model is the special case when $M$ is the empty setoid (no atoms).

We say that a setoid $M=\left(|M|,=_{M}\right)$ belongs to the universe $U$ if there is some $m: U$ with $|M|=T(m)$, and some $e:|M| \longrightarrow|M| \longrightarrow U$ such that for all $x, y:|M|$,

$$
x==_{M} y \Longleftrightarrow T(e(x, y)) \text {. }
$$

For such setoids we have:

Lemma 5.1. If $M$ is a setoid which belongs to $U$, then the relations $x==_{V} y$ and $x \in_{V} y$ are propositions in $U$.

It is crucial that the basic relations $\in$ and $=$ are interpreted as propositions in the universe $U$ in order to be able to verify that all bounded formulas ( $\Delta_{0}$-formulas) may be used in the separation scheme of CZF. We will thus consider $V(M)$ where the setoid $M$ belongs to $U$. There is no principal difficulty in extending the construction to finitely many setoids of atoms.

5.2. $V$-representable setoids. We consider here for simplicity only pure sets, thus let $V=V(\emptyset)$. For each $u: V$ define the setoid

$$
B(u)=\left(|B(u)|,=_{B(u)}\right)
$$

of elements of $V$ belonging to $u$ by letting

$$
|B(u)|=\Sigma z: V . z \in_{V} u
$$

and

$$
(z, p)=_{B(u)}\left(z^{\prime}, p^{\prime}\right) \Longleftrightarrow z={ }_{V} z^{\prime} .
$$

Note that for a set $u=\sup (a, f)$, it holds that

$$
B(\sup (a, f)) \cong\left(T(a), \sim_{f}\right)
$$

where

$$
x \sim_{f} x^{\prime} \Longleftrightarrow f(x)={ }_{V} f\left(x^{\prime}\right) .
$$


We define therefore

$$
R(\sup (a, f))=\left(T(a), \sim_{f}\right) .
$$

It is thereby easy to find the setoid and its underlying type from the set. A setoid $A$ is $V$-representable iff there is some $u: V$ and a bijection $\phi: A \cong R(u)$. Let $u=\sup (a, f)$ and $v=\sup (b, g)$. If we examine

$$
\operatorname{Ext}(R(u), R(v))
$$

the standard construction of the setoid of functions from $R(u)$ to $R(v)$, it has the underlying type

$$
\Sigma h: T(a) \longrightarrow T(b) .\left(\forall x, y: T(a)\left(f x={ }_{V} f y \Rightarrow g(h x)=_{V} g(h y)\right)\right)
$$

and equality $\sim$ defined by

$$
(h, p) \sim\left(h^{\prime}, p^{\prime}\right) \text { iff } \forall x: T(a) \cdot g(h x)={ }_{V} g\left(h^{\prime} x\right) .
$$

Let $F_{u, v}$ denote the type in (5.2). Define

$$
\gamma(h, p)=\sup (a, \lambda x \cdot\langle f x, g(h x)\rangle)
$$

which gives the graph of the function $h$, when $(h, p): F_{u, v}$. Suppose that the type $F_{u, v}$ has a code $\varphi_{u, v}$ in $U$ so that $F_{u, v}=T\left(\varphi_{u, v}\right)$. Now we can form

$$
v^{u}=\sup \left(\varphi_{u, v}, \gamma\right)
$$

which is the set all of functions from $u$ to $v$. Indeed we have

$$
z \in_{V} v^{u} \text { iff } z \text { is a total and functional relation from } u \text { to } v,
$$

where the latter can be formally expressed as the conjunction of the following statements

$$
\begin{gathered}
(\forall t \in V)\left(t \in_{V} z \Rightarrow(\exists x, y \in V)\left(x \in_{V} u \wedge y \in_{V} v \wedge t=_{V}\langle x, y\rangle\right)\right), \\
(\forall x \in V)\left(x \in_{V} u \Rightarrow(\exists y \in V)\left(y \in_{V} v \wedge\langle x, y\rangle \in_{V} z\right)\right), \\
\left(\forall x, y, y^{\prime} \in V\right)\left(\langle x, y\rangle \in_{V} z \wedge\left\langle x, y^{\prime}\right\rangle \in_{V} z \Rightarrow y=_{V} y^{\prime}\right) .
\end{gathered}
$$

Note that these are the interpretations of the corresponding first-order CZF formulas in the structure $\left(V,=_{V}, \in_{V}\right)$. We have the following bijective correspondence

Proposition 5.2. For any $u=\sup (a, f), v=\sup (b, g) \in V$, there is a bijection

$$
\psi: R\left(v^{u}\right) \longrightarrow \operatorname{Ext}(R(u), R(v))
$$

given by $\psi(h, p)=(h, p)$.

Actually we have arrived at the standard definition of the function set by analyzing representable sets and functions. 
5.3. Two isomorphic categories. The internal category of sets in $V$ may be described as follows. Define the category $\mathcal{V}$ to have as objects $\mathcal{V}_{0}$ the setoid $V=\left(V,=_{V}\right)$. The arrows $\mathcal{V}_{1}$ has as underlying type

$$
\Sigma u \in V \text {.Isarrow }(u)
$$

where $\operatorname{Isarrow}(u)$ is the predicate definable using CZF formulas

$$
\exists a, b, f \in V \cdot u=V\langle\langle a, b\rangle, f\rangle \wedge f \text { is a total and functional relation from } a \text { to } b .
$$

Equality $(u, p)=\mathcal{V}_{1}\left(u^{\prime}, p^{\prime}\right)$ is defined to be $u={ }_{V} u^{\prime}$. The setoid $\mathcal{V}_{2}$ of composable arrows has for underlying type

$$
\Sigma w \in V . \Sigma u, v \in \mathcal{V}_{1} \cdot w={ }_{V}\left\langle\pi_{1}(u), \pi_{1}(v)\right\rangle \wedge \operatorname{cod} u={ }_{v} \operatorname{dom} v
$$

and its equality is given by $(w, p) \sim\left(w^{\prime}, p^{\prime}\right)$ iff $w={ }_{V} w^{\prime}$. Again these can be given by straightforward intepretations of first-order CZF-formulas. Composition $\mathrm{cmp}$ of arrows is obtained by composition of relations in the usual set-theoretic way.

Theorem 5.3. $\mathcal{V}$ is a category.

A different category is constructed using the method of Section 4.1. We extend $R(\cdot)$ to a family of setoids $\bar{R}$ over the setoid $V=\left(V,=_{V}\right)$. Let $\bar{R}(\alpha)=R(\alpha)$ for $\alpha \in V$. For a proof object $p$ for $\sup (a, f)=_{V} \sup (b, g)$, or equivalently, for

$$
\forall x: T(a) . \exists y: T(b) . f(x)={ }_{V} g(y) \wedge \forall y: T(b) . \exists x: T(a) . f(x)=_{V} g(y),
$$

we thus have

$$
\forall x: T(a) \cdot f(x)=_{V} g\left(\pi_{1}\left(\pi_{1}(p)(x)\right)\right) \text { and } \forall y: T(b) . f\left(\pi_{1}\left(\pi_{2}(p)(y)\right)\right)=_{V} g(y) .
$$

Let $\bar{R}(p)(x)=\pi_{1}\left(\pi_{1}(p)(x)\right)$. This defines an extensional function

$$
\bar{R}(p): R(\sup (a, f)) \longrightarrow R(\sup (b, g)) .
$$

Lemma 5.4. $\bar{R}$ is a family of setoids over $\left(V,=_{V}\right)$.

Proof. The function $\bar{R}(p): \bar{R}(\sup (a, f)) \longrightarrow \bar{R}(\sup (b, g))$, is independent of $p$. Indeed, if $p, p^{\prime}$ are arbitrary and $x \sim_{f} x^{\prime}$, then

$$
g(\bar{R}(p)(x))=_{V} f(x)=_{V} f\left(x^{\prime}\right)=_{V} g\left(\bar{R}\left(p^{\prime}\right)(x) .\right.
$$

This verifies (F2). If $p: \sup (a, f)=_{V} \sup (a, f)$, then $f(\bar{R}(p)(x))=_{V} f(x)$, so $\bar{R}(p)(x) \sim_{f} x$. Hence $\bar{R}(p)$ is the identity, and (F1) is clear. Finally, we check (F3'). Suppose we have three proof objects $p: \sup (a, f)=_{V} \sup (b, g), q: \sup (b, g)=_{V} \sup (c, h)$ and $r: \sup (a, f)=_{V}$ $\sup (c, h)$. Expanding as above we have $g(\bar{R}(p)(x))=_{V} f(x)$ and $h(\bar{R}(q)(y))=_{V} g(y)$ for all $x$ and $y$. Thus

$$
h(\bar{R}(q)(\bar{R}(p)(x)))=_{V} g(\bar{R}(p)(x))={ }_{V} f(x)
$$

for all $x$. Now the third proof object gives similarly $h(\bar{R}(r)(x))=_{V} f(x)$ for all $x$. Hence for all $x$,

$$
\bar{R}(q)(\bar{R}(p)(x)) \sim_{h} \bar{R}(r)(x) .
$$

Thus $\bar{R}$ is a family of setoids over $\left(V,=_{V}\right)$. 
From the family $(V, \bar{R})$, we may construct the category $\mathcal{C}=\mathcal{C}(V, \bar{R})$, as in Section 4.1 and, then compare it to the category $\mathcal{V}$ above. The objects of the two categories are give by the same setoid. Let $F_{0}: \mathcal{C}_{0} \longrightarrow \mathcal{V}_{0}$ be the identity map. There is a bijection $\mathcal{C}_{1} \longrightarrow \mathcal{V}_{1}$ given by

$$
(a, b, f) \mapsto\left\langle\langle a, b\rangle, \gamma\left(|f|, \operatorname{ext}_{f}\right)\right\rangle .
$$

Further, this yields a bijection $F_{2}: \mathcal{C}_{2} \longrightarrow \mathcal{V}_{2}$ by letting $F_{1}$ act on the two component arrows. It is then straightforward to verify that $F_{0}, F_{1}$ and $F_{2}$ form a functor which is an isomorphism. We have

Theorem 5.5. The categories $\mathcal{C}(V, \bar{R})$ and $\mathcal{V}$ are isomorphic.

5.4. CZFU - constructive sets with urelements. The model $V(M)$ in Section 5.1 suggests an axiomatization of CZF with urelements or atoms. For an example of a classical set theory with atoms, see e.g. [14]. In [1], a theory called $\mathrm{CZF}^{\mathrm{I}}$, which is CZF extended with a class of individuals, is mentioned but the axioms are not detailed in that paper. It is not clear to us whether it is actually a version of the theory presented below. Nevertheless, we propose the following axiomatization of CZF with atoms, CZFU.

The language is that of set theory, with a binary predicate for membership $\in$, extended with unary predicate $\mathrm{S}$, for being a set. Define $\mathrm{A}(x)=\neg \mathrm{S}(x)$. Write $\forall^{\mathrm{S}} x \ldots$ for $\forall x . \mathrm{S}(x) \Rightarrow \ldots$ and $\exists^{\mathrm{S}} x \ldots$ for $\exists x . \mathrm{S}(x) \wedge \ldots$

The axioms are the following

(C1) $\forall x . \mathrm{S}(x) \vee \mathrm{A}(x)$. Each object is either a set or an atom.

(C2) $\forall x y . y \in x \Rightarrow \mathrm{S}(x)$. An object which has an element must be a set.

(C3) $\forall^{\mathrm{S}} x \cdot \forall^{\mathrm{S}} y \cdot(\forall z . z \in x \Longleftrightarrow z \in y) \Rightarrow x=y$. Sets are determined by their elements.

(C4) Let $\varphi(x)$ be any formula. Then take set-induction for this formula as an axiom

$$
(\forall x .(\forall y \in x . \varphi(x)) \Rightarrow \varphi(x)) \Rightarrow \forall x \cdot \varphi(x) .
$$

Since atoms have no elements this is actually equivalent to

$$
(\forall x . \mathrm{A}(x) \Rightarrow \varphi(x)) \Rightarrow\left(\forall^{\mathrm{S}} x \cdot(\forall y \in x \cdot \varphi(x)) \Rightarrow \varphi(x)\right) \Rightarrow \forall x \cdot \varphi(x) .
$$

(C5) Union: $\forall^{\mathrm{S}} x \cdot \exists^{\mathrm{S}} u \cdot(\forall z . z \in u \Longleftrightarrow(\exists y \in x) z \in y)$.

(C6) Pairing: $\forall x y \cdot \exists^{\mathrm{S}} u .(\forall z . z \in u \Longleftrightarrow(z=x \vee z=y))$.

(C7) Bounded separation: Let $\varphi(x)$ be any bounded formula. Then take as an axiom:

$$
\forall^{\mathrm{S}} u \cdot \exists^{\mathrm{S}} v \cdot \forall x \cdot x \in v \Longleftrightarrow x \in u \wedge \varphi(x) \text {. }
$$

(C8) Subset collection: for any formula $\varphi$

$$
\begin{aligned}
\forall a b . \exists^{\mathrm{S}} c . \forall u . & (\forall x \in a . \exists y \in b . \varphi(x, y, u)) \Rightarrow \\
& \exists d \in c .(\forall x \in a . \exists y \in d . \varphi(x, y, u)) \wedge(\forall y \in d . \exists x \in a . \varphi(x, y, u))
\end{aligned}
$$

(C9) Strong collection: for any formula $\varphi$

$\forall a .(\forall x \in a . \exists y . \varphi(x, y)) \Rightarrow \exists^{S} b .(\forall x \in a . \exists y \in b . \varphi(x, y)) \wedge(\forall y \in b . \exists x \in a . \varphi(x, y))$

(C10) Infinity axiom:

$$
\exists^{\mathrm{S}} x . \emptyset \in x \wedge(\forall y \in x) y^{+} \in x .
$$

Here $y^{+}=\{y,\{y\}\}$. 
If we add the purity axiom (everything is a set) we get a system, which is easily seen to be equivalent to the standard CZF.

$$
\text { (Purity): } \forall x . S(x) \text {. }
$$

Theorem 5.6. For any setoid $M=\left(|M|,=_{M}\right)$ belonging to $U$, the set-theoretic universe $V(M)$ is a model of CZFU. The model also verifies that there is a set containing all atoms, that is

$$
\exists^{\mathrm{S}} x . \forall z . z \in x \Longleftrightarrow \mathrm{A}(z) .
$$

Proof. The proof is similar to the verification in Aczel's standard set-theoretic model in case of the axioms $\mathrm{C} 3-\mathrm{C} 6, \mathrm{C} 8-\mathrm{C} 10$. The axioms $\mathrm{C} 1$ and $\mathrm{C} 2$ are directly verified by the meaning of $\mathrm{A}$ and $\epsilon_{V}$. As for axiom $\mathrm{C} 7$, bounded separation, we may use the standard proof once we have noticed that by Lemma 5.1, $a=_{V} b$ and $a \in_{V} b$ are in $U$, whenever $M$ is in $U$.

To verify (5.3) first construct $a=\sup (m, f)$ where $m: U$ is such that $T(m)=M$ and $f: M \longrightarrow V$ is given by $f(t)=\operatorname{atom}(t)$. Then for any $z \in V, z \in_{V} a$ if, and only if, there is $t: T(m)$ such that $z={ }_{V}$ atom $(t)$, that is $\mathrm{A}(t)$ is true.

\section{The implementation in Coq And applications}

In our Coq implementation [17] we understand setoids in the sense of propositions-as-types, which means that the equality relation takes its truth values in Set or Type. This is in contrast to the standard setoids of Coq where the equality relation is Prop-valued. (Cf. total setoids of Barthe et al. [3].) We have used the built-in type set to interpret the universe $U$. The setoids belonging to $U$ are therefore setoids based on Set and called just setoids. What we call setoids in this paper is called Typeoid in the Coq code and they are based on Type.

The $V$-sets and $V(M)$-sets are constructed using the generalized inductive definitions available for Type of Coq. They could as well have been constructed using a general W-type. In several places record types are used, which corresponds to $\Sigma$-type applications of MLTT. The following theorems of the paper are formalized: Theorems 4.2, 4.4.(d), 4.5, 5.3, 5.5, and 5.6 .

We verify as well the Regular Extension Axiom (REA) 2] in our Coq implementation. This axiom is crucial for formalizing transfinite inductive definitions in CZF. There are important extensions of the REA [9] that unfortunately seem difficult to model in the Coqsystem, since the system currently lacks the ability to handle general inductive-recursive definition.

Apart from using a set-theoretic universe to overcome the limitations of the built in equality of intensional type theory, there are also possible practical utilizations in proof development. An application of the kind of implementation presented here is to integrate type-theoretic and set-theoretic methods in proofs. The set-theoretic methods make it possible avoid certain coherence problems that may be difficult to solve in type theory, for instance regarding families of setoids and involved inductive definitions. The type-theoretic

methods have the well known advantages with type checking that guide construction of proofs, and a direct computational interpretation. One may develop theorems in CZF (or $\mathrm{CZFU}$ ) and then translate the first order formulas and proofs into the richer language that 
is modelled in the Coq implementation. This translation can easily be done automatically, and the development of the CZF theorems could be done in a theorem prover or proof assistant that can handle intuitionistic logic.

\section{REFERENCES}

[1] Peter Aczel. The type-theoretic interpretation of constructive set theory. In: A. Macintyre, L. Pacholski and J. Paris (eds.), Logic Colloquium 'ry. North-Holland, Amsterdam 1978.

[2] Peter Aczel. The type-theoretic interpretation of constructive set theory: inductive definitions. In: R.B. Marcus, G.J. Dorn, and G.J.W. Dorn (eds.), Logic, Methodology, and Philosophy of Science VII, NorthHolland, Amsterdam and New York, 1986, pp. $17-49$.

[3] Gilles Barthe, Venanzio Capretta and Olivier Pons. Setoids in type theory. Journal of Functional Programming 13(2003), pp. 261-293.

[4] Benno van den Berg and Ieke Moerdijk. A unified approach to Algebraic Set Theory. In: S.B. Cooper, H. Geuvers, A. Pillay and J. Väänänen (eds.) Logic Colloquium 2006, Lecture Notes in Logic, Cambridge University Press 2009, pp. 18 - 37.

[5] Aurelio Carboni. Some free constructions in realizability and proof theory. Journal of Pure and Applied Algebra 103 (1995), pp. $117-148$.

[6] Jason J. Hickey. The MetaPRL Logical Programming Environment. PhD thesis, Cornell University, Ithaca, NY, January 2001.

[7] Martin Hofmann. On the Interpretation of Type Theory in Locally Cartesian Closed Categories In: Proceedings of Computer Science Logic, Lecture Notes in Computer Science, Springer, 1994, pp. 427 441.

[8] Martin Hofmann and Thomas Streicher. The groupoid interpretation of type theory. In: G. Sambin and J. Smith (eds.) Twenty-five years of constructive type theory (Venice, 1995), pp. 83 - 111, Oxford Logic Guides, 36, Oxford Univ. Press, New York, 1998.

[9] Robert S. Lubarsky and Michael Rathjen. On the regular extension axiom and its variants. Mathematical Logic Quarterly 49 (2003), pp. 513 - 518.

[10] Saunders Mac Lane. Categories for the Working Mathematician. 2nd ed. Springer 1997.

[11] Maria E. Maietti. Modular correspondences between dependent type theories and categorical universes including pretopoi and topoi. Mathematical Structures in Computer Science 15 (2005), pp. 1089 -1149.

[12] Nax P. Mendler. Note: An Implementation of Constructive Set Theory in the LEGO System. Department of Computer Science, Manchester University 1991.

[13] Ieke Moerdijk and Erik Palmgren. Type Theories, Toposes and Constructive Set Theory: Predicative Aspects of AST. Annals of Pure and Applied Logic 114(2002), pp. 155 - 201.

[14] Yannis N. Moschovakis. Notes on Set Theory. Second Edition. Springer 2006.

[15] Erik Palmgren. Proof-relevance of families of setoids and identity in type theory. Archive for Mathematical Logic 51(2012), pp. $35-47$.

[16] Erik Palmgren. Constructivist and Structuralist Foundations: Bishop's and Lawvere's Theories of Sets. Annals of Pure and Applied Logic 163(2012), pp. 1384 - 1399.

[17] Erik Palmgren and Olov Wilander. www.math.su.se/ palmgren/coq/czf_and_setoids File repository of the implementation described in the present paper.

[18] Thomas Streicher. Investigations into intensional type theory. Habilitation Thesis, Ludwig-Maximilians Universität, Munich, 1993. http://www.mathematik.tu-darmstadt.de/ ${ }^{\text {streicher/ }}$

[19] Makoto Takeyama. Personal communication.

[20] Olov Wilander. Constructing a small category of setoids. Mathematical Structures in Computer Science 22(2012), pp. $103-121$.

[21] Xin Yu. Formalizing abstract algebra in constructive set theory. Master's thesis, California Institute of Technology, 2002. 